\title{
Luminescent Lead Halide Ionic Liquids for High-Spatial-Resolution Fast Neutron Imaging
}

\author{
Viktoriia Morad, ${ }^{\#}$ Kyle M. McCall," Kostiantyn Sakhatskyi, Eberhard Lehmann, Bernhard Walfort, \\ Adrian S. Losko, Pavel Trtik, Markus Strobl, Sergii Yakunin, and Maksym V. Kovalenko*
}

Cite This: ACS Photonics 2021, 8, 3357-3364

Read Online

\section{ACCESS | Lill Metrics \& More | 回 Article Recommendations ｜（） Supporting Information}

ABSTRACT: The fast neutron imaging technique with recoil proton detection harbors significant potential for imaging of thick, large-scale objects containing high- $Z$ elements. However, the challenge to find efficient fast neutron scintillators with high spatial resolution is ongoing. The list of requirements for such scintillators is long and demanding: a proton-rich, scattering-free material combining high light yield with the absence of light reabsorption. To meet these challenges, we look for a suitable material among a rising class of $\mathrm{OD}$ organic-inorganic $\mathrm{Pb}$ (II) halide hybrids. The use of large organic cations, e.g., trihexyltetradecylphosphonium, results in room-temperature ionic liquids that combine highly Stokes-shifted (up to $1.7 \mathrm{eV}$ ), reabsorption-free, and efficient

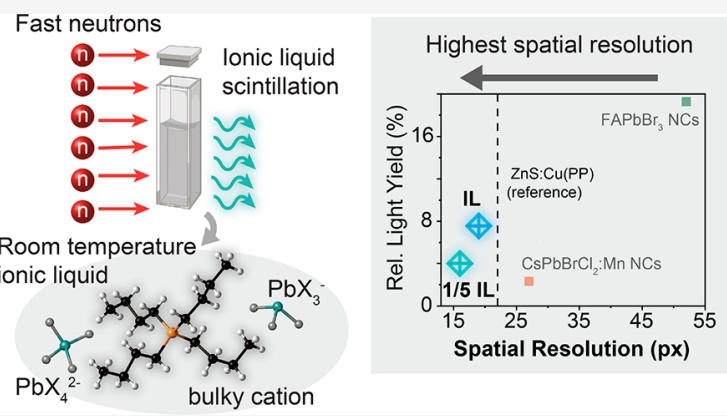
emission (photoluminescence quantum yield up to 60\%) from molecularly small and dense $\left(\mathrm{PbX}_{2}\right.$ molar fraction up to 0.33$)$ emitting centers. We investigate the optical properties of the resulting ionic liquids and showcase their utility as fast neutron imaging scintillators. Concomitantly with good light yield, such fast-neutron scintillators exhibit both higher spatial resolution and lower $\gamma$-ray sensitivity compared with commercial ZnS:Cu-based screens.

KEYWORDS: ionic liquid, fast neutrons, scintillation, imaging, lead halides

\section{INTRODUCTION}

As opposed to thermal neutrons with relatively low penetration depth or high-energy X-rays with insufficient low-Z element contrast, fast neutrons $(1-15 \mathrm{MeV})$ are the tool of choice for imaging thick objects containing both high- and low-Z elements. ${ }^{1}$ The primary method for fast neutron detection is based on elastic scattering of neutrons by nuclei, generating recoil nuclei (typically protons) with very small penetration depths (up to tens of micrometers) in the detector material. The kinetic energy of these recoil nuclei is then deposited as ionized charge carriers in the detector material, which can excite a scintillator to emit visible-range photons detectable by conventional imaging devices such as a CCD camera. However, the potential of this method depends on the exploration of efficient scintillator materials. The perfect scintillator for this indirect detection scheme should be a scattering-free, proton-rich, and high-light-yield material with a dense concentration of (preferably atomically small) fast emitting centers.

As the-state-of-art fast neutron scintillators face their own challenges, ${ }^{2}$ researchers start to divert their course toward $n s^{2}$ metal halides, ${ }^{3}$ a rapidly expanding class of optoelectronic materials with promising applications in high-energy detection. ${ }^{4}$ Vast structural versatility within this class results in a plethora of materials, which can be roughly divided into semiconductors (3D corner-sharing $\mathrm{ABX}_{3}$ and related $2 \mathrm{D}$ structures $)^{5,6}$ and electronically and structurally lower-dimensional compounds (non-corner-sharing 2D, 1D, and isolated $0 \mathrm{D}){ }^{7,8}$ For instance, colloidal $\mathrm{ABX}_{3}$ nanocrystals (NCs) dispersed in a proton-rich medium (organic solvents) make for a suitable scintillation medium; ${ }^{3}$ however, their strong selfabsorption (small Stokes shift) limits achievable spatial resolution and light yield. Zero-dimensional metal halides, due to their molecular-like largely Stokes-shifted emission, are particularly successful in indirect detection schemes as X-ray scintillators. $^{9-12}$

Herein, we explore $0 \mathrm{D} \mathrm{Pb}(\mathrm{II})$ metal halides as scintillators for fast neutron detection and imaging. Zero-dimensional $\mathrm{Pb}$ (II) halides are typically composed of isolated anionic $\mathrm{PbX}_{n}$ centers (mono- or polynuclear) ${ }^{10,13}$ and inorganic (e.g., $\left.\mathrm{Cs}^{+}\right)^{14,15}$ or organic cations. The emission originates from electron and hole pairs trapped on metal halide species ${ }^{16}$ and can be tuned in the UV-to-green spectral range by halide $(\mathrm{Cl}$ or $\mathrm{Br})$ and coordination number $(3,4,6) .{ }^{17,18}$ The reabsorption-free quality of their emission renders a good

Received: September 3, 2021

Published: October 25, 2021 
a Ionic Liquids of lead(II) halides

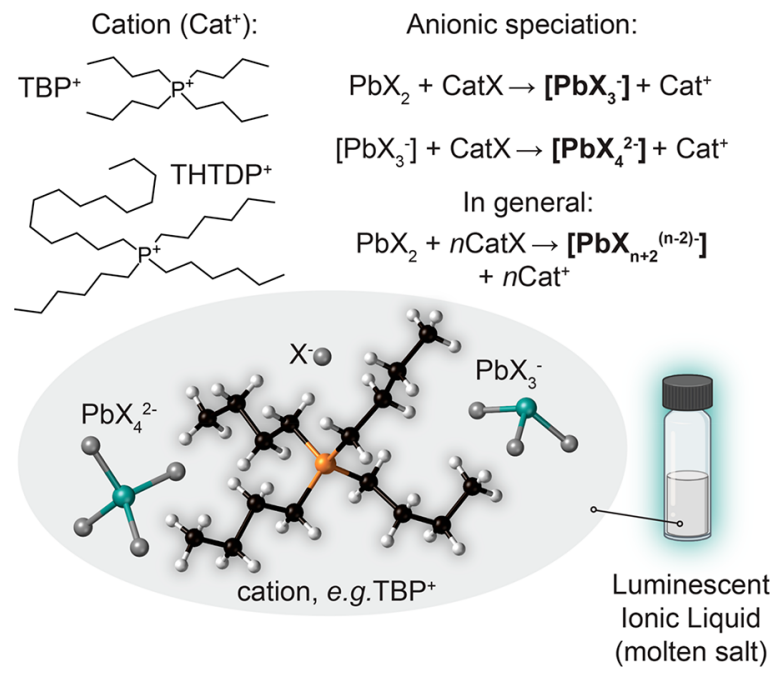

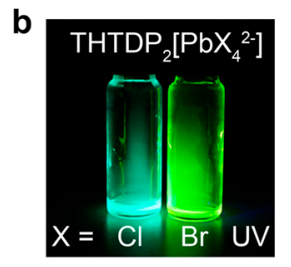
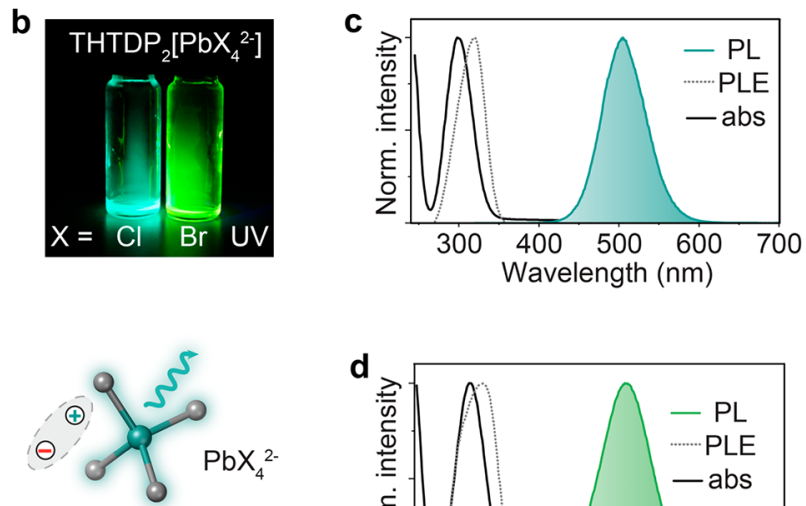

Molecular-like emission

$\rightarrow$ large Stokes shift

$\rightarrow$ narrow excitation $\rightarrow$ broad PL

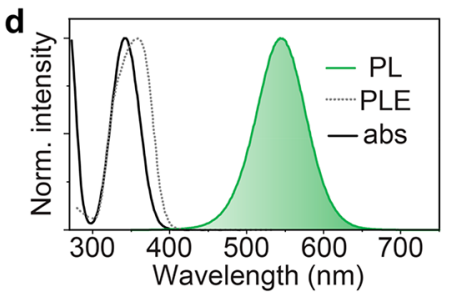

Figure 1. (a) Building blocks of haloplumbate(II) ILs: cationic species used in this study include TBP and THTDP phosphonium cations, while anionic speciation depends on the molar ratio between $\mathrm{Pb}$ and $\mathrm{X}$ (where $\mathrm{X}=\mathrm{Cl}, \mathrm{Br}$ ). (b) Photo of the THTDPX-PbX $(\mathrm{X}=\mathrm{Cl}, \mathrm{Br}$ ) samples displaying typical $\mathrm{PbX}_{4}{ }^{2-}$ emission under $\mathrm{UV}(365 \mathrm{~nm}$ ) excitation. PL, PLE (dashed line) and absorption (solid line) spectra of the (c) 3THTDPCl-PbCl $\mathrm{Pb}_{2}$ and (d) $3 \mathrm{THTDPBr}-\mathrm{PbBr}_{2}$.

prerequisite for high spatial resolution in fast neutron imaging. However, the majority of OD materials are crystalline bulk ionic salts. Among approaches to achieve scattering-free formulations of $\mathrm{ns}^{2} \mathrm{OD}$ materials are colloidal NCs, which are thus far limited to fully inorganic compositions, such as $\mathrm{OD}$ $\mathrm{Cs}_{4} \mathrm{PbBr}_{6}$ (nonluminescent), ${ }^{19} \mathrm{Cs}_{4} \mathrm{SnX}_{6}$ (luminescent but airsensitive), ${ }^{20}$ and others. ${ }^{21,22}$ Another strategy would be to prevent the crystallization of $\mathrm{OD}$ salts, preferably at room temperature (RT). Such supercooled liquid materials are known as RT molten salts or ionic liquids (ILs). ${ }^{23}$ To form an $\mathrm{IL}$, large organic ionic species are required: their bulkiness reduces the degree of ion association, which is reflected in a depressed melting point. These organic cations make also for an ideal proton-rich medium required for interactions with neutrons and subsequent conversion of the recoil-proton energy into luminescence from the anionic component. Herein we show that luminescent ILs derived from $\mathrm{PbX}_{2}$ function as efficient fast neutron scintillators. The inherently high luminescence Stokes-shift translates into high spatial resolution, superior to the commercial $\mathrm{ZnS}$ : $\mathrm{Cu}$-based screens in the reference experiments.

\section{RESULTS AND DISCUSSION}

Halometallate ILs comprising metal halide anions and large organic cations (e.g., alkylmethylimidazolium $\left(\mathrm{C}_{n} \mathrm{mim}\right)$, tetraalkylammonium, tetraalkylphosphonium, etc.) are particularly common and span across most metals in the periodic table. $^{24}$ The functionality of halometallate IL is typically defined by the properties of its anionic species, and catalytic, ${ }^{25}$ magnetic, ${ }^{26}$ and luminescent ${ }^{27}$ ILs have been reported. For the present study, we have chosen tetraalkylphosphonium cations, namely, tetrabutylphosphonium (TBP) and trihexyltetradecylphosphonium (THTDP) to pair with lead(II) halide, yielding luminescent ILs. The choice was partially motivated by a previous study on chloroplumbate(II) IL speciation, ${ }^{28}$ which demonstrated that in the THTDPCl- $\mathrm{PbCl}_{2}$ system the solidus line lies below $-60{ }^{\circ} \mathrm{C}$. The haloplumbate(II) ILs have been prepared by dissolving the required amount of $\mathrm{PbX}_{2}$ in molten
THTDPX (at RT) or TBPX (at $T>70^{\circ} \mathrm{C}$ ). Synthetic details can be found in the Methods section. Figure la depicts the main building blocks of the resulting ILs. According to a prior study, $\left[\mathrm{PbX}_{4}{ }^{2-}\right]$ species are present across the whole $\mathrm{PbX}_{2}$ molar ratio range $\left(0.55 \geq \chi_{\mathrm{PbX}_{2}} \geq 0.05\right)$, while $\left[\mathrm{PbX}_{3}{ }^{-}\right]$were only detected for $\chi_{\mathrm{PbX} 2} \geq 0.33$. $^{28}$

Optical Properties of Lead(II) Halide ILs. Figure $1 \mathrm{~b}$ demonstrates representative lead(II) chloride and bromide ILs with the $\mathrm{THTDP}^{+}$cation, for a cation to lead(II) halide ratio of 3:1 or molar ratio $\chi_{\mathrm{PbX} 2}=0.25$. The maximum molar ratio $\chi_{\mathrm{PbX} 2} \geq 0.33$ is also achievable; however, the samples with such high loadings are viscous and pose inconveniences during their handling. ${ }^{28}$ It has to be noted that although there exists a study on the speciation in THTDPCl- $\mathrm{PbCl}_{2}$ system, ${ }^{28}$ no optical properties of these species have been investigated. Both halide systems feature largely Stokes-shifted $(200 \mathrm{~nm})$ and broad (60-80 nm) blue-green photoluminescence (PL) and sharp, molecular-like UV PL excitation (PLE); for the chloride, both PL and PLE are shifted toward higher energies (Figure 1c,d). Such optical properties are very characteristic of self-trapped OD metal halide centers. ${ }^{10,13,29}$ Moreover, optical properties of ILs closely resemble previously studied species formed when lead(II) halides are dissolved in polar solvents (e.g., $\mathrm{N}, \mathrm{N}$ dimethylformamide, dimethyl sulfoxide, acetonitrile, polyethylene glycole) with an excess of halide anions provided by quaternary ammonium salts. ${ }^{18,30,31}$ Typically, the absorption maximum shifts toward lower energies with the increase of coordination number in the order $\mathrm{PbX}_{2}>\mathrm{PbX}_{3}{ }^{-}>\mathrm{PbX}_{4}{ }^{2-}$. 32,33 However, we have found that the PL and PLE properties of the ILs change little in the compositional range from $0.09 \leq \chi_{\mathrm{PbX} 2}$ $\leq 0.25$ (Table S1). The big difference in RT PL quantum yield (PLQY) between chloride and bromide ILs (60\% vs 5\%) can be attributed to more efficient phonon-exciton PL quenching for heavier $\mathrm{Br}$, as has been observed for other $\mathrm{OD} \mathrm{ns}^{2}$-metal halides. ${ }^{34}$ On the basis of previous research, we tentatively assign the luminescence observed in samples with $\chi_{\mathrm{PbX} 2} \leq 0.33$ to $\left[\mathrm{PbX}_{4}{ }^{2-}\right]$ species. In extremely diluted samples $\left(\chi_{\mathrm{PbX} 2}=\right.$ $0.0025)$, a blue-shifted PL peak around $420 \mathrm{~nm}$ starts to appear 
along with $\mathrm{PbX}_{4}^{2-} \mathrm{PL}$, which can be attributed to species with higher coordination number, e.g., $\mathrm{PbX}_{6}^{4-}$ (Figure $\mathrm{S} 1$ ).

Fast Neutron Imaging with Lead(II) Halide ILs. Protonrich cations in the ILs are well suited for the indirect fast neutron detection scheme, depicted in Figure 2a. The high

a Recoil proton detection with Ionic Liquids

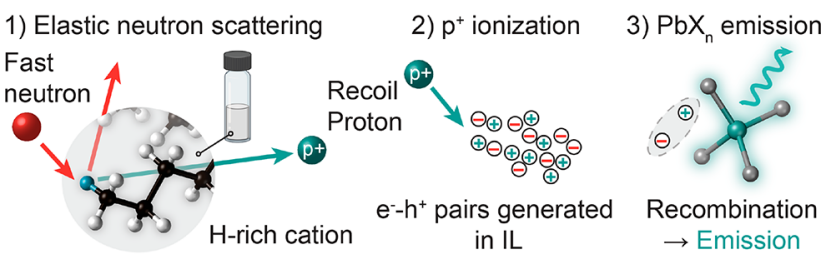

b Ionic Liquid scintillators for fast neutron imaging

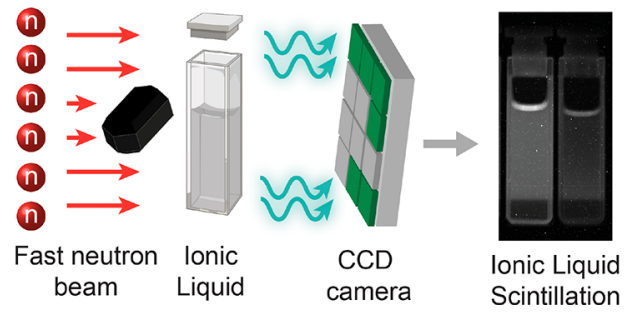

Figure 2. (a) Schematics of the processes involved in the recoil proton detection with ILs: (1) elastic neutron scattering on the H-rich cation with the generation of a recoil proton, (2) ionization of the recoil proton with subsequent generation of the electron-hole pairs in IL, and (3) recombination of the electron-hole pairs and emission of visible light photon. (b) Scheme of the indirect fast neutron imaging experiment with ILs as a scintillator. energy recoil proton, generated by the scattering of the fast neutron off the cation, generates electron-hole pairs, which in turn recombine on the emissive species with the generation of visible light photons. Fast neutron imaging in such a scheme can be easily realized by introducing a CCD camera that detects the emission behind the scintillator ILs, as depicted in Figure $2 \mathrm{~b}$. Lead(II) halide ILs combine attractive features of prospective scintillators for fast neutron imaging: ${ }^{35-37}$ intimately mixed highly emissive molecular phosphors and long organic chains to provide sufficient stopping power for fast neutrons. ${ }^{3}$ Furthermore, the transparency of these interdissolved materials eliminates the phosphor-plastic interface, which scatters light in the commercial $\mathrm{ZnS}: \mathrm{Cu}(\mathrm{PP})$ phosphor screens, while their short-lived emission lifetimes (450 ns, Figure S2) also stand in stark contrast to the minuteslong afterglow effects observed in $\mathrm{ZnS}: \mathrm{Cu}(\mathrm{PP})$ screens. ${ }^{2}$ Most importantly, the large Stokes shifts of these emitters minimize self-absorption losses in light yield and eliminates photon recycling (reabsorption and emission) that would reduce spatial resolution.

Light Output of Lead(II) Halide ILs in a Fast Neutron Imaging Experiment. A representative composition THTDPCl- $\mathrm{PbCl}_{2}\left(3: 1\right.$ or $\left.\chi_{\mathrm{PbX} 2}=0.25\right)$ (referred below as "IL") and that diluted $1 / 5$ with neat THTDPCl ("1/5-IL") were selected for fast neutron imaging measurements. These measurements were conducted at the NECTAR ${ }^{38}$ beamline of the FRM-II research reactor (Garching, Germany). The details of the beamline and experimental setup have been described previously. ${ }^{39,3}$ In short, the sample cuvettes are sealed inside a light-tight scintillation chamber with a CCD camera that detects the radioluminescence produced by the cuvettes upon fast neutron irradiation.
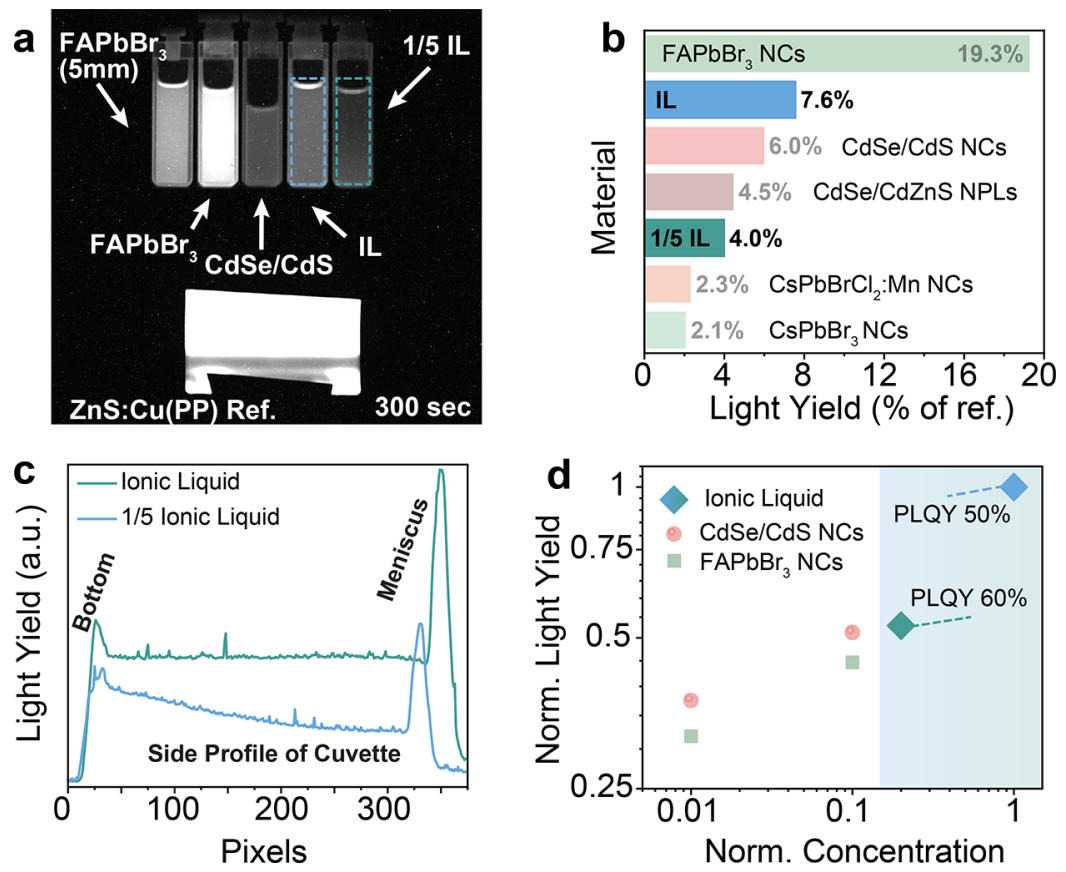

Figure 3. (a) Radiograph of IL under fast neutron irradiation (300 s exposure) as compared with nanocrystal scintillators ${ }^{3}$ and a commercial $\mathrm{ZnS}: \mathrm{Cu}(\mathrm{PP})$ screen, used here as a reference. (b) Total light yield comparison of an ionic liquid with leading fast neutron NC scintillators, ${ }^{3}$ given as a percentage of the light yield obtained for the reference $\mathrm{ZnS}: \mathrm{Cu}(\mathrm{PP})$ scintillator screen under identical conditions (300 s exposure, full concentration, 10-mm-thick cuvettes). (c) Light yield of concentrated and dilute IL vs vertical pixel position from the radiograph in panel (a), showing the light outcoupling at the cuvette edge and meniscus, as well as the imperfect mixing of the diluted sample leading to a higher $\mathrm{PbCl}_{2}$ concentration at the bottom of the sample. (d) Concentration-dependent light yield of IL and selected NC scintillators. ${ }^{3}$ 

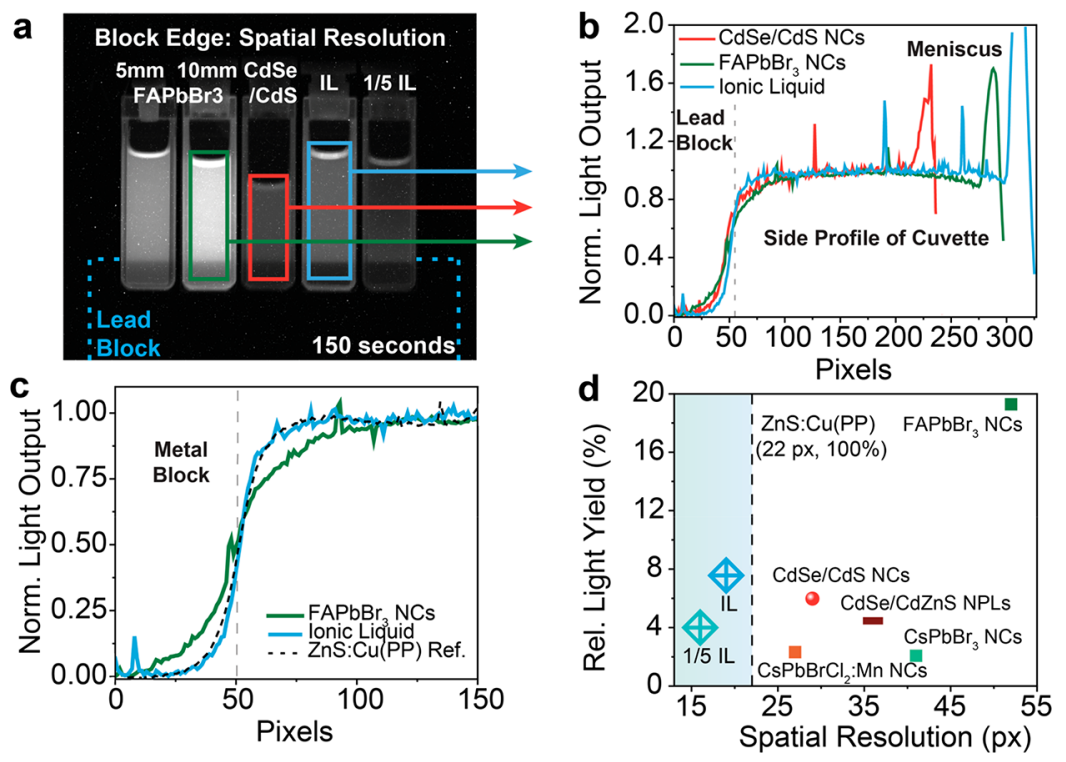

Figure 4. (a) Radiograph of IL and colloidal $\mathrm{NCs}^{40}$ with a lead block in the beam for a sharp edge to measure spatial resolution, $150 \mathrm{~s}$ fast neutron beam exposure. (b) Side profile of the light output from the ionic liquid cuvette, with the unattenuated fast neutron beam normalized to 1 , while the 0 is set to the dark value of the lead-blocked beam, including the side profiles of $\mathrm{CdSe} / \mathrm{CdS}$ and $\mathrm{FAPbBr}_{3} \mathrm{NCs}^{40}$ for comparison. (c) Zoomedin side profile comparison with the edge of the $\mathrm{ZnS}: \mathrm{Cu}(\mathrm{PP})$ reference, highlighting the better resolution of the ionic liquid. (d) Summary of fast neutron imaging characteristics of these ionic liquids and colloidal $\mathrm{NCs}^{40}$ with the high Stokes shift ionic liquid providing superior resolution due to reduced scattering. Panels (a) and (b) are adapted from ref 40. Copyright 2020 American Chemical Society.

A series of comparative radiographs of the ionic liquid samples and some colloidal $\mathrm{NCs},{ }^{3}$ along with a commercial $\mathrm{ZnS}: \mathrm{Cu}(\mathrm{PP})$ screen, were measured under fast neutron exposure for times ranging from 30 to $300 \mathrm{~s}$ (e.g., Figure 3a). The results clearly demonstrate the proof-of-concept for fast neutron detection in ILs, with bright radioluminescence from these samples under exposure to a fast neutron beam (Figure 3a). The light yield of the ionic liquid is linear with increasing exposure time and reproducible across multiple series of such measurements (Figure S3), indicating the absence of degradation or long-lived afterglow effects. Note that imperfect mixing of the $1 / 5$-diluted ionic liquid (1/5-IL) (done on-site) results in an uneven light yield across the height of the cuvette (side profile rotated for clarity, Figure $3 \mathrm{c}$ ).

The total light yield is reported as a percentage of the total light yield of the reference $\mathrm{ZnS}: \mathrm{Cu}(\mathrm{PP})$ scintillator under the same conditions, and we find that the light yields of the IL and $1 / 5$-IL are $7.6 \%$ and $4.0 \%$ of the reference, respectively (Figure $3 \mathrm{~b})$. The light yields of IL and 1/5-IL are competitive with semiconductor $\mathrm{NC}$ colloids $\left(\mathrm{FAPbBr}_{3}, \mathrm{CdSe} / \mathrm{CdS} \mathrm{NCs}\right.$ and NPLs, $\mathrm{CsPbBrCl} 2: \mathrm{Mn}, \mathrm{CsPbBr}_{3}$ ) as scintillators, which we introduced previously, ${ }^{3}$ despite higher PLQYs of these NCs (up to 90\%). This is likely the result of the large Stokes shift of the IL sample, which limits self-absorption losses that plague many of the NC scintillators. The significantly higher phosphor loading of these materials is also beneficial, with the undiluted IL (3:1) having a $25 \%$ mass loading, close to the $30 \%$ of the $\mathrm{ZnS}: \mathrm{Cu}(\mathrm{PP})$ reference. The high light yield of this IL demonstrates the proof-of-concept for IL-based neutron scintillators pairing emissive metal centers with large organics.

The reduced light yield of the 1/5-IL compared to the undiluted IL indicates a concentration-dependent loss of efficiency (Figure $3 c$ and Figure S4), as the PLQY of the IL and $1 / 5$-IL samples are similar in this concentration range ( $50 \%$ and $60 \%$, respectively), and thus a reduction in PLQY is not responsible for the lower light yield. This indicates that the charge conversion efficiency (CCE), or the proportion of ionized charge carriers that are sufficiently close to emissive centers to induce light output, ${ }^{3}$ is reduced at lower concentration because the ionized electron cloud generated by the recoil proton interacting with fewer luminescent lead(II) centers. This trend was previously observed also for NC colloids as scintillators, ${ }^{3}$ which also found that higher concentrations are beneficial for enhanced light yield and thus showed that the ideal concentration for unity CCE is not achieved (Figure $3 \mathrm{~d}$ ). ${ }^{3}$ In the present case, which is a 10 -fold increase in the mass loading (25 mass \%) relative to the NC colloids (e.g., 2.1 mass \% for $\left.\mathrm{FAPbBr}_{3} \mathrm{NCs}\right)^{3}$ and is on par with the ideal loading observed for $\mathrm{ZnS}: \mathrm{Cu}(\mathrm{PP})$, this may indicate that there is a proportion of lead(II) metal centers that are nonemissive and act as parasitic absorbers of charge carriers, degrading the PLQY and lowering the observed CCE.

To examine this possibility and test whether even higher concentrations could improve upon this light yield, a follow-up study was conducted at the NEUTRA ${ }^{39}$ neutron imaging beamline of the Paul Scherrer Institut (PSI), Switzerland. In this test, the same IL samples (1/5-IL and IL) were compared with a new sample of higher concentration (denoted Conc-IL, $\chi_{\mathrm{PbX} 2}=0.33$ ). Note that this experiment was carried out over 9 months after the initial experiments at FRM-II, evidencing the stability of these materials.

The Conc-IL sample showed a discoloration due to absorption in the visible range (Figure S5), and accordingly the maximal light yield was achieved with the "IL" sample $\chi_{\mathrm{PbX} 2}$ $=0.25$ (Figure S6). This indicates that the chemistry of the TTDPCl- $\mathrm{PbCl}_{2}$ system changes at high concentrations, with the addition of parasitic absorption that reduces the light yield under fast neutron irradiation. Nevertheless, the high light yield of this IL despite the possible presence of parasitic centers demonstrates the benefits of IL-based scintillators with significantly higher phosphor concentrations than that achievable with NCs. 
Spatial Resolution of Lead(II) Halide ILs in a Fast Neutron Imaging Experiment. Turning to test the spatial resolution of the IL-based scintillator, a lead block $(2.5 \mathrm{~cm}$ thick) is placed in the path of the fast neutron beam to provide a sharp edge to the transmitted beam, leading to light (unblocked) and dark (attenuated) regions in the scintillator (Figure 4a). The spatial resolution is then estimated by comparing the sharpness of the edge in the scintillator's change in light yield across this region, as shown in the side profile of the cuvette (rotated for clarity, Figure $4 \mathrm{~b}$ ). Here, we utilize the $10-90 \%$ rise as a conservative numeric measure of the edge sharpness, normalizing the light and dark regions to 1 and 0 light output, respectively, and measuring the number of pixels needed to go from 0.1 to 0.9 (each pixel represents $\sim 0.1 \mathrm{~mm}$ ).

The brightest $\mathrm{NCs}\left(\mathrm{FAPbBr}_{3}\right.$ and $\left.\mathrm{CdSe} / \mathrm{CdS}\right)$ are included for comparison, and the undiluted IL exhibits significantly better spatial resolution than both NCs.

The edge measured with the IL is slightly sharper than that of the $\mathrm{ZnS}: \mathrm{Cu}(\mathrm{PP})$ reference (Figure $4 \mathrm{c}$ ). The edge width of the IL is 19 pixels, far below the values of 52 and 29 pixels for $\mathrm{FAPbBr}_{3}$ and $\mathrm{CdSe} / \mathrm{CdS} \mathrm{NCs}$, respectively, and even better than the $\mathrm{ZnS}: \mathrm{Cu}(\mathrm{PP})$ reference at 22 pixels (Figure $4 \mathrm{~d}$, Table S2). The $1 / 5$-IL is lower still at 16 pixels, though the signal-tonoise ratio is reduced due to lower light yield.

The impressive spatial resolution observed for the IL highlights the critical role of self-transparency in obtaining high-spatial-resolution scintillators, whereas the NCs exhibit absorption and re-emission of scintillation light that obscures the sharp edge, clearly observed in $\mathrm{FAPbBr}_{3}$ with the emission tail extending throughout the dark region (Figure $4 \mathrm{~b}$ ). This result also demonstrates that the lack of a scattering phosphor-plastic interface can yield improvements even over the commercial standard $\mathrm{ZnS}: \mathrm{Cu}(\mathrm{PP})$. Furthermore, the $\mathrm{ZnS}: \mathrm{Cu}(\mathrm{PP})$ phosphor screen $(2.4 \mathrm{~mm})$ is less than a quarter of the thickness of the IL cuvette $(10 \mathrm{~mm})$, because the resolution of such screens has been shown to decrease as thickness rises, forcing a trade-off between light yield and spatial resolution. ${ }^{41}$ In contrast, the large Stokes shift of the lead(II)-based IL exhibits excellent resolution despite thicker screens, allowing for scaled-up screens of arbitrary thickness to maximize the detector efficiency (and thereby light yield) without sacrificing spatial resolution.

An important criterion for a prospective fast neutron scintillator is the $\gamma$-ray sensitivity. For instance, the uranium fission reaction used at NECTAR to generate the fast neutron beam also produces a high $\gamma$-ray flux, which contaminates the fast neutron image if the phosphor is sensitive also to $\gamma$-rays. This is detrimental to the performance, as such effects must be counteracted by shielding the $\gamma$-ray contribution with heavy absorbers, thereby reducing the fast neutron flux. The blocking experiment provides a way to evaluate the contribution of $\gamma$ rays to the observed scintillation response: the lead block changes the light yield and has known neutron and $\gamma$-ray attenuation coefficients $a_{\mathrm{n}}$ and $a_{\gamma}$. Thus, the ratio of the light yield in the light and dark regions of the IL, $R_{\text {bright }}$ can be used to derive the ratio $R_{\mathrm{n} / \gamma}$ of light yield induced by neutrons and $\gamma$-rays for a metal block with thickness $d:{ }^{40}$

$$
R_{\mathrm{n} / \gamma}=\frac{\mathrm{e}^{-d a_{\gamma}}-R_{\text {bright }}}{R_{\text {bright }}-\mathrm{e}^{-d a_{\mathrm{n}}}}
$$

The $\gamma$-ray attenuation coefficient of lead was averaged with respect to the prompt $\gamma$-ray spectrum of the ${ }^{235} \mathrm{U} \gamma$-ray spectrum. ${ }^{42}$ The calculated neutron fission spectrum was used to estimate the fast neutron attenuation coefficient of the lead block. ${ }^{43}$ Note that this overestimates the neutron stopping power because the calculated average energy $(1.35 \mathrm{MeV})$ is less than the actual average energy delivered at NECTAR (1.8 $\mathrm{MeV}),{ }^{38}$ underestimating the neutron contribution to the light yield and thereby overestimating the $\gamma$-ray sensitivity.

The light yield ratio $R_{\mathrm{n} / \gamma}$ for the IL is $1.76(5)$, well above the reported value of $1.0-1.1$ for $\mathrm{ZnS}: \mathrm{Cu}(\mathrm{PP})$ as measured on the same beamline. ${ }^{2}$ This means that $64 \%$ of the scintillation light is derived from neutrons for the $\mathrm{IL}$, while that of $\mathrm{ZnS}: \mathrm{Cu}(\mathrm{PP})$ is only $50-52 \%$, enhancing the neutron-specific light yield of the IL to $9.7 \%$ of the $\mathrm{ZnS}: \mathrm{Cu}(\mathrm{PP})$ reference. Dilution further reduces the $\gamma$-ray sensitivity, as the $1 / 5$-IL exhibits an $R_{\mathrm{n} / \gamma}$ of 1.97(5). While these values are not so high as the ratios obtained previously for the NCs $(2.2-4.1),{ }^{40}$ they are promising values considering the higher density of the concentrated IL relative to the dilute NCs. For reference, over $14 \mathrm{~cm}$ of $\mathrm{Pb}$ shielding is needed to lower the $\gamma$-ray contribution to $1 \%$ of the signal due to the low $R_{\mathrm{n} / \gamma}$ of $\mathrm{ZnS}: \mathrm{Cu}(\mathrm{PP})$, while an $R_{\mathrm{n} / \gamma}$ of 1.7 reduces this to only $9.6 \mathrm{~cm}$ of $\mathrm{Pb}^{41}$ This permits substantially higher fluxes to be used and drastically shortens collection times; to put this improvement in context, eliminating $4.4 \mathrm{~cm}$ of $\mathrm{Pb}$ shielding is equivalent to eliminating 1.8 of the $2.5-\mathrm{cm}$-thick $\mathrm{Pb}$ blocks used here to provide the contrast in Figure 4a. This allows for a much higher fast neutron flux and benefits tomography applications where reconstruction tasks are too calculation-heavy to remove the $\gamma$-ray-induced "background" signal.

\section{CONCLUSIONS}

In conclusion, this work demonstrates the proof-of-concept for IL-based fast neutron scintillators and provides guidelines to identify promising IL-metal halide compositions. $\mathrm{Pb}$ (II) halide ILs display a compelling set of characteristics required for fast neutron imaging: they are scattering-free, include proton-rich cations, and permit a high density of the emitting centers. These qualities, together with high PLQY (60\%) and large Stokes shift (up to $1.7 \mathrm{eV}$ ), result in a spatial resolution superior to the established $\mathrm{ZnS}: \mathrm{Cu}$ scintillators. Future work should build upon these advances by improving the PLQY of ILs and achieving a higher concentration of emissive species.

\section{METHODS}

Materials. Trihexyltetradecylphosphonium chloride (THTDPCl, $>95 \%$, IoLiTec), trihexyltetradecylphosphonium bromide (THTDPBr, $>95 \%$, IoLiTec), tetrabutylphosphonium chloride ( $\mathrm{TBPCl}, 95 \%, \mathrm{ABCR}$ ), tetrabutylphosphonium bromide (TBPBr, $\geq 98 \%$, Fluka), lead(II) chloride $\left(\mathrm{PbCl}_{2}\right.$, 99.999\%, metal basis, $\mathrm{ABCR})$, and lead(II) bromide $\left(\mathrm{PbBr}_{2}\right.$, 99.999\%, metal basis, ABCR) were used.

Preparation of Lead(II) Halide ILs. The list of chemicals and their purity can be found in the Supporting Information. All chemicals were used as received without further purification. ILs and lead halide salts were stored in an $\mathrm{N}_{2}$ filled glovebox to avoid moisture, as the neat ionic liquids are hygroscopic. In a typical synthetic procedure of $n$ THTDP$\mathrm{PbX}_{2}, \mathrm{PbCl}_{2}$ or $\mathrm{PbBr}_{2}$ powders were mixed with a required volume of THTDPCl or THTDPBr, correspondingly. To aid the dissolution of lead halides, suspensions were stirred with a magnetic stirrer and heated to around $100-120{ }^{\circ} \mathrm{C}$ on a hot plate. In such conditions, samples with $\chi_{\mathrm{PbX} 2} \leq 0.25$ can be 
prepared in about $30 \mathrm{~min}$, while samples with $\chi_{\mathrm{PbX} 2} \geq 0.33$ require overnight stirring. For the $n \mathrm{TBPX}-\mathrm{PbX}_{2}$, wherein TBPX are solids, alkyl phosphonium halides and lead halides were mixed in the desired ratio and heated to about 100-120 ${ }^{\circ} \mathrm{C}$ on a hot plate. After several minutes, the TBPX salts melted and $\mathrm{PbX}_{2}$ dissolution continued upon stirring. The neat ILs were stored in the glovebox for further analysis and experiments.

PL and PLE steady-state spectra were recorded either with FluoroMax4-Plus-P (Horiba Jobin Yvon) or Fluorolog 3 (Horiba Jobin Yvon), equipped with 150 and $500 \mathrm{~W}$ xenon lamps, respectively.

UV-vis absorbance spectra were collected on a Jasco V670 spectrophotometer equipped with a deuterium (D2) lamp $(190-350 \mathrm{~nm})$ for use in UV, a halogen lamp $(330-2700 \mathrm{~nm})$ for use in UV/NIR, and an integrating sphere (ILN-725) with a working wavelength range of $220-2200 \mathrm{~nm}$. Ionic liquids were drop cast between two quartz slides.

PL decay traces were recorded with a FluoTime 300 spectrometer from PicoQuant Gmbh equipped with a TimeHarp 260 PICO counting unit. As en excitation source for time-resolved PL traces, a frequency-tripled Nd:YAGlaser (wavelength of $355 \mathrm{~nm}$, a power density of about $10 \mathrm{~mW} \mathrm{~cm}^{-2}$, pulse duration $10 \mathrm{ps}$ ) was used. A scattering light from excitation was suppressed on the entrance of the emission monochromator by a $400 \mathrm{~nm}$ long-pass filter. The samples were measured in a quartz cuvette with a $10 \mathrm{~mm}$ path length.

Absolute PLQYs were measured using a Quantaurus-QY spectrometer from Hamamatsu.

Fast Neutron Imaging at NECTAR. Fast neutron imaging was conducted at the FRM-II reactor (Garching, Germany) on the NECTAR (neutron computed tomography and radiography) beamline. ${ }^{38}$ A schematic of the instrument and a thorough description of the experimental setup have been provided previously. ${ }^{40}$ In short, the setup includes a sample stage to place materials for imaging and a light-tight scintillator enclosure with a CCD camera (CCD ANDOR iKon-L BV camera) $;^{44}$ the collimation ratio L/D was set to 200 . For fast neutron imaging tests, the scintillator enclosure was sealed with a thin aluminum plate, to which a cuvette sample holder was attached with aluminum tape. The scintillator samples were placed into cuvettes and sealed to the enclosure with aluminum tape. Light yield measurements were taken of an empty sample stage, while thick metal blocks (either $1.5-\mathrm{cm}$ thick $\mathrm{W}, 5-\mathrm{cm}$-thick $\mathrm{Fe}$, or 2.5 -cm-thick $\mathrm{Pb}$ ) were placed in the beam path to provide a sharp edge for spatial resolution measurements. Note that a pixel represents approximately 0.1 $\mathrm{mm}$ with the current camera focus, determined by the width of the $10 \mathrm{~mm}$ cuvettes at 103 pixels $(0.097 \mathrm{~mm} /$ pixel $)$. Images were processed using the Fiji distribution ${ }^{45}$ of Image J. ${ }^{46}$

Fast Neutron Imaging at NEUTRA (PSI). A follow-up fast neutron imaging experiment to test the light yield of the highest concentration IL sample was conducted at the NEUTRA beamline of the Swiss spallation neutron source at the Paul Scherrer Institute (Villigen, Switzerland). Briefly, the cuvettes with the ionic liquids and the reference $\mathrm{ZnS}: \mathrm{Cu}$ plastic scintillator were placed in the light-tight camera box (MIDI-box camera detector, CCD ANDOR iKon-L camera). The camera box was placed at the measuring position 2 (approximately $7.3 \mathrm{~m}$ downstream from the beam-defining aperture) of the NEUTRA beamline; thus the collimation ratio $\mathrm{L} / \mathrm{D}$ was set to 365 . In order to screen out the thermal neutron contribution, light yield measurements were taken with the experimental shutter closed (composed of $\mathrm{B}_{4} \mathrm{C}$ and $\mathrm{Cd}$ layers) while the high energy and fail-safe shutters were open. An additional $2 \mathrm{~mm} \mathrm{Cd}$ filter was inserted in the beam path at approximately $3.8 \mathrm{~m}$ downstream of the beam-defining aperture to further attenuate the thermal neutrons. The images were taken with $120 \mathrm{~s}$ exposure times with the original pixel size corresponding to $\sim 103 \mu \mathrm{m}$. To reduce the noise, the images were binned by a factor of $4 \times 4$, resulting in the pixel size of $\sim 412 \mu \mathrm{m}$. Images were processed using the $\mathrm{Fiji}^{45}$ distribution of ImageJ. ${ }^{46}$

\section{ASSOCIATED CONTENT}

\section{Supporting Information}

The Supporting Information is available free of charge at https://pubs.acs.org/doi/10.1021/acsphotonics.1c01348.

PL decay plot of the IL sample, light yield vs time plot, spatial resolution of dilute and concentrated ionic liquid, comparison of absorption spectra of Conc-IL and IL, PSI NEUTRA beamline fast neutron data, summary tables for optical properties and neutron detection characteristics (PDF)

\section{AUTHOR INFORMATION}

\section{Corresponding Author}

Maksym V. Kovalenko - Laboratory of Inorganic Chemistry, Department of Chemistry and Applied Biosciences, ETH Zürich, CH-8093 Zürich, Switzerland; Laboratory for Thin Films and Photovoltaics, Empa - Swiss Federal Laboratories for Materials Science and Technology, CH-8600 Dübendorf, Switzerland; 이이.org/0000-0002-6396-8938; Email: mvkovalenko@ethz.ch

\section{Authors}

Viktoriia Morad - Laboratory of Inorganic Chemistry, Department of Chemistry and Applied Biosciences, ETH Zürich, CH-8093 Zürich, Switzerland; Laboratory for Thin Films and Photovoltaics, Empa - Swiss Federal Laboratories for Materials Science and Technology, CH-8600 Dübendorf, Switzerland

Kyle M. McCall - Laboratory of Inorganic Chemistry, Department of Chemistry and Applied Biosciences, ETH Zürich, CH-8093 Zürich, Switzerland; Laboratory for Thin Films and Photovoltaics, Empa - Swiss Federal Laboratories for Materials Science and Technology, CH-8600 Dübendorf, Switzerland; 이이.orcid.org/0000-0001-8628-3811

Kostiantyn Sakhatskyi - Laboratory of Inorganic Chemistry, Department of Chemistry and Applied Biosciences, ETH Zürich, CH-8093 Zürich, Switzerland; Laboratory for Thin Films and Photovoltaics, Empa - Swiss Federal Laboratories for Materials Science and Technology, CH-8600 Dübendorf, Switzerland

Eberhard Lehmann - Paul Scherrer Institut, 5232 Villigen PSI, Switzerland

Bernhard Walfort - RC Tritec Ltd., 9053 Teufen, Switzerland

Adrian S. Losko - Forschungs-Neutronenquelle Heinz MaierLeibnitz, 85748 Garching, Germany

Pavel Trtik - Paul Scherrer Institut, 5232 Villigen PSI, Switzerland

Markus Strobl - Paul Scherrer Institut, 5232 Villigen PSI, Switzerland

Sergii Yakunin - Laboratory of Inorganic Chemistry, Department of Chemistry and Applied Biosciences, ETH 
Zürich, CH-8093 Zürich, Switzerland; Laboratory for Thin Films and Photovoltaics, Empa - Swiss Federal Laboratories for Materials Science and Technology, CH-8600 Dübendorf, Switzerland; ○ orcid.org/0000-0002-6409-0565

Complete contact information is available at: https://pubs.acs.org/10.1021/acsphotonics.1c01348

\section{Author Contributions \\ ${ }^{\#}$ V.M. and K.M.M. contributed equally. \\ Notes}

The authors declare no competing financial interest.

\section{ACKNOWLEDGMENTS}

This work was financially supported by the European Union through the Horizon 2020 Research and Innovation Programme (ERC CoG Grant, grant agreement no. 819740, project SCALE-HALO), by the Swiss National Science Foundation (grant agreement no. 188404), by RC Tritec AG, and by ETH Zurich through the ETH+ Project SynMatLab. This work is based upon experiments performed at the NECTAR instrument operated by FRM II at the Heinz Maier-Leibnitz Zentrum (MLZ), Garching, Germany. This work is based on experiments performed at the NEUTRA beamline of the Swiss spallation neutron source SINQ Paul Scherrer Institute, Villigen, Switzerland. The authors acknowledge fruitful discussions with Dr. Yevhen Shynkarenko. The authors gratefully acknowledge the support of R. Schütz, the NECTAR beamline engineer for experimental setup, as well as Dipl. Ing. Armin Kriele for on-site chemistry laboratory support.

\section{REFERENCES}

(1) Dangendorf, V.; Zboray, R. Potential and Status in Imaging with Fast Neutrons. Neutron News 2015, 26 (2), 27-30.

(2) Makowska, M. G.; Walfort, B.; Zeller, A.; Grunzweig, C.; Bucherl, T. Performance of the Commercial PP/ZnS:Cu and PP/ $\mathrm{ZnS}: \mathrm{Ag}$ Scintillation Screens for Fast Neutron Imaging. J. Imaging 2017, 3 (4), 60.

(3) McCall, K. M.; Sakhatskyi, K.; Lehmann, E.; Walfort, B.; Losko, A. S.; Montanarella, F.; Bodnarchuk, M. I.; Krieg, F.; Kelestemur, Y.; Mannes, D.; Shynkarenko, Y.; Yakunin, S.; Kovalenko, M. V. Fast Neutron Imaging with Semiconductor Nanocrystal Scintillators. ACS Nano 2020, 14 (11), 14686-14697.

(4) Kakavelakis, G.; Gedda, M.; Panagiotopoulos, A.; Kymakis, E.; Anthopoulos, T. D.; Petridis, K. Metal Halide Perovskites for HighEnergy Radiation Detection. Adv. Sci. 2020, 7, 2002098.

(5) Stoumpos, C. C.; Kanatzidis, M. G. The Renaissance of Halide Perovskites and Their Evolution as Emerging Semiconductors. Acc. Chem. Res. 2015, 48 (10), 2791-2802.

(6) Saparov, B.; Mitzi, D. B. Organic-Inorganic Perovskites: Structural Versatility for Functional Materials Design. Chem. Rev. 2016, 116 (7), 4558-4596.

(7) Smith, M. D.; Connor, B. A.; Karunadasa, H. I. Tuning the Luminescence of Layered Halide Perovskites. Chem. Rev. 2019, 119 (5), 3104-3139.

(8) Lin, H.; Zhou, C.; Tian, Y.; Siegrist, T.; Ma, B. Low-Dimensional Organometal Halide Perovskites. ACS Energy Lett. 2018, 3 (1), 5462.

(9) He, Q. Q.; Zhou, C. K.; Xu, L. J.; Lee, S. J.; Lin, X. S.; Neu, J.; Worku, M.; Chaaban, M.; Ma, B. W. Highly Stable Organic Antimony Halide Crystals for X-ray Scintillation. ACS Mater. Lett. 2020, 2 (6), 633-638.

(10) Morad, V.; Shynkarenko, Y.; Yakunin, S.; Brumberg, A.; Schaller, R. D.; Kovalenko, M. V. Disphenoidal Zero-Dimensional Lead, Tin, and Germanium Halides: Highly Emissive Singlet and
Triplet Self-Trapped Excitons and X-ray Scintillation. J. Am. Chem. Soc. 2019, 141 (25), 9764-9768.

(11) Xu, L.-J.; Lin, X.; He, Q.; Worku, M.; Ma, B. Highly Efficient Eco-Friendly X-ray Scintillators Based on an Organic Manganese Halide. Nat. Commun. 2020, 11 (1), 4329.

(12) Lian, L. Y.; Zheng, M. Y.; Zhang, W. Z.; Yin, L. X.; Du, X. Y.; Zhang, P.; Zhang, X. W.; Gao, J. B.; Zhang, D. L.; Gao, L.; Niu, G. D.; Song, H. S.; Chen, R.; Lan, X. Z.; Tang, J.; Zhang, J. B. Efficient and Reabsorption-Free Radioluminescence in $\mathrm{Cs}_{3} \mathrm{Cu}_{2} \mathrm{I}_{5}$ Nanocrystals with Self-Trapped Excitons. Adv. Sci. 2020, 7 (11).

(13) Zhou, C. K.; Lin, H. R.; Neu, J.; Zhou, Y.; Chaaban, M.; Lee, S.; Worku, M.; Chen, B. H.; Clark, R.; Cheng, W. H.; Guan, J. J.; Djurovich, P.; Zhang, D. Z.; Lu, X. J.; Bullock, J.; Pak, C.; Shatruk, M.; Du, M. H.; Siegrist, T.; Ma, B. W. Green Emitting Single-Crystalline Bulk Assembly of Metal Halide Clusters with Near-Unity Photoluminescence Quantum Efficiency. ACS Energy Lett. 2019, 4 (7), $1579-1583$.

(14) Nikl, M.; Mihokova, E.; Nitsch, K. Photoluminescence \& Decay Kinetics of $\mathrm{Cs}_{4} \mathrm{PbCl}_{6}$ Single Crystals. Solid State Commun. 1992, 84 (12), 1089-1092.

(15) Nikl, M.; Mihokova, E.; Nitsch, K.; Somma, F.; Giampaolo, C.; Pazzi, G. P.; Fabeni, P.; Zazubovich, S. Photoluminescence of $\mathrm{Cs}_{4} \mathrm{PbBr}_{6}$ crystals and thin films. Chem. Phys. Lett. 1999, 306 (5), 280-284.

(16) Han, D.; Shi, H. L.; Ming, W. M.; Zhou, C. K.; Ma, B. W.; Saparov, B.; Ma, Y. Z.; Chen, S. Y.; Du, M. H. Unraveling Luminescence Mechanisms in Zero-Dimensional Halide Perovskites. J. Mater. Chem. C 2018, 6 (24), 6398-6405.

(17) Bohun, A.; Dolejší, J.; Barta, Č. The Absorption and Luminescence of $\left(\mathrm{PbCl}_{6}\right)^{4-}$ and $\left(\mathrm{PbBr}_{6}\right)^{4-}$ Complexes. Czech. J. Phys. 1970, 20 (7), 803-807.

(18) Nikol, H.; Becht, A.; Vogler, A. Photoluminescence of Germanium(II), Tin(II), and Lead(II) Chloride Complexes in Solution. Inorg. Chem. 1992, 31 (15), 3277-3279.

(19) Akkerman, Q. A.; Park, S.; Radicchi, E.; Nunzi, F.; Mosconi, E.; De Angelis, F.; Brescia, R.; Rastogi, P.; Prato, M.; Manna, L. Nearly Monodisperse Insulator $\mathrm{Cs}_{4} \mathrm{PbX}_{6}(\mathrm{X}=\mathrm{Cl}, \mathrm{Br}, \mathrm{I})$ Nanocrystals, Their Mixed Halide Compositions, and Their Transformation into $\mathrm{CsPbX}_{3}$ Nanocrystals. Nano Lett. 2017, 17 (3), 1924-1930.

(20) Tan, L.; Wang, W.; Li, Q.; Luo, Z.; Zou, C.; Tang, M.; Zhang, L.; He, J.; Quan, Z. Colloidal Syntheses of Zero-Dimensional $\mathrm{Cs}_{4} \mathrm{SnX}_{6}$ $(\mathrm{X}=\mathrm{Br}, \mathrm{I})$ Nanocrystals with High Emission Efficiencies. Chem. Commun. 2020, 56 (3), 387-390.

(21) Cheng, P.; Sun, L.; Feng, L.; Yang, S.; Yang, Y.; Zheng, D.; Zhao, Y.; Sang, Y.; Zhang, R.; Wei, D.; Deng, W.; Han, K. Colloidal Synthesis and Optical Properties of All-Inorganic Low-Dimensional Cesium Copper Halide Nanocrystals. Angew. Chem., Int. Ed. 2019, 58 (45), 16087-16091.

(22) Wang, A.; Yan, X.; Zhang, M.; Sun, S.; Yang, M.; Shen, W.; Pan, X.; Wang, P.; Deng, Z. Controlled Synthesis of Lead-Free and Stable Perovskite Derivative $\mathrm{Cs}_{2} \mathrm{SnI}_{6}$ Nanocrystals via a Facile Hot-Injection Process. Chem. Mater. 2016, 28 (22), 8132-8140.

(23) MacFarlane, D. R. K.; Pringle, J. M. An Introduction to Ionic Liquids. In Fundamentals of Ionic Liquids; Wiley-VCH Verlag $\mathrm{GmbH}$ \& Co. KGaA: Weinheim, Germany, 2017; pp 1-25.

(24) Estager, J.; Holbrey, J. D.; Swadzba-Kwasny, M. Halometallate Ionic Liquids - Revisited. Chem. Soc. Rev. 2014, 43 (3), 847-886.

(25) Kore, R.; Berton, P.; Kelley, S. P.; Aduri, P.; Katti, S. S.; Rogers, R. D. Group IIIA Halometallate Ionic Liquids: Speciation and Applications in Catalysis. ACS Catal. 2017, 7 (10), 7014-7028.

(26) Yoshida, Y.; Saito, G. Design of Functional Ionic Liquids Using Magneto- and Luminescent-Active Anions. Phys. Chem. Chem. Phys. 2010, 12 (8), 1675-1684.

(27) Alvarez-Vicente, J.; Dandil, S.; Banerjee, D.; Gunaratne, H. Q. N.; Gray, S.; Felton, S.; Srinivasan, G.; Kaczmarek, A. M.; Van Deun, R.; Nockemann, P. Easily Accessible Rare-Earth-Containing Phosphonium Room-Temperature Ionic Liquids: EXAFS, Luminescence, and Magnetic Properties. J. Phys. Chem. B 2016, 120 (23), 53015311. 
(28) Coleman, F.; Feng, G.; Murphy, R. W.; Nockemann, P.; Seddon, K. R.; Swadzba-Kwasny, M. Lead(II) Chloride Ionic Liquids and Organic/Inorganic Hybrid Materials - a Study of Chloroplumbate(II) Speciation. Dalton T. 2013, 42 (14), 5025-5035. (29) Zhou, C. K.; Lin, H. R.; Worku, M.; Neu, J.; Zhou, Y.; Tian, Y.; Lee, S.; Djurovich, P.; Siegrist, T.; Ma, B. Blue Emitting Single Crystalline Assembly of Metal Halide Clusters. J. Am. Chem. Soc. 2018, 140 (41), 13181-13184.

(30) Oldenburg, K.; Vogler, A. Electronic-Spectra and Photochemistry of Tin(II), Lead(II), Antimony(III), and Bismuth(III) Bromide Complexes in Solution. Z. Naturforsch., B: J. Chem. Sci. 1993, 48 (11), 1519-1523.

(31) Chin, S.-H.; Choi, J. W.; Hu, Z.; Mardegan, L.; Sessolo, M.; Bolink, H. J. Tunable Luminescent Lead Bromide Complexes. J. Mater. Chem. C 2020, 8 (45), 15996-16000.

(32) Byrne, R. H.; Yao, W. S.; Luo, Y. X.; Millero, F. J. Complexation of $\mathrm{Pb}(\mathrm{II})$ by Chloride Ions in Aqueous Solutions. Aquat. Geochem. 2010, 16 (3), 325-335.

(33) Stamplecoskie, K. G.; Manser, J. S.; Kamat, P. V. Dual Nature of the Excited State in Organic-Inorganic Lead Halide Perovskites. Energy Environ. Sci. 2015, 8 (1), 208-215.

(34) Morad, V.; Yakunin, S.; Kovalenko, M. V. Supramolecular Approach for Fine-Tuning o the Bright Luminescence from ZeroDimensional Antimony(III) Halides. Acs Mater. Lett. 2020, 2 (7), $845-852$.

(35) Zboray, R.; Adams, R.; Morgano, M.; Kis, Z. Qualification and Development of Fast Neutron Imaging Scintillator Screens. Nucl. Instrum. Methods Phys. Res., Sect. A 2019, 930, 142-150.

(36) Buffler, A.; Tickner, J. Detecting Contraband Using Neutrons: Challenges and Future Directions. Radiat. Meas. 2010, 45 (10), $1186-1192$.

(37) Kardjilov, N.; Manke, I.; Woracek, R.; Hilger, A.; Banhart, J. Advances in Neutron Imaging. Mater. Today 2018, 21 (6), 652-672.

(38) Bücherl, T.; Söllradl, S. NECTAR: Radiography and Tomography Station Using Fission Neutrons. JLSRF 2015, 1, A19.

(39) Lehmann, E. H.; Vontobel, P.; Wiezel, L. Properties of the Radiography Facility NEUTRA at SINQ and its Potential for Use as European Reference Facility. Nondestruct. Test. Nondestr. Test. Eval. 2001, 16 (2-6), 191-202.

(40) McCall, K. M.; Sakhatskyi, K.; Lehmann, E.; Walfort, B.; Losko, A. S.; Montanarella, F.; Bodnarchuk, M. I.; Krieg, F.; Kelestemur, Y.; Mannes, D.; Shynkarenko, Y.; Yakunin, S.; Kovalenko, M. V. Fast Neutron Imaging with Semiconductor Nanocrystal Scintillators. ACS Nano 2020, 14, 14686.

(41) Makowska, M. G.; Walfort, B.; Zeller, A.; Grünzweig, C.; Bücherl, T. Performance of the Commercial PP/ZnS: $\mathrm{Cu}$ and PP/ $\mathrm{ZnS}$ : Ag Scintillation Screens for Fast Neutron Imaging. J. Imaging 2017, 3 (4), 60.

(42) Makii, H.; Nishio, K.; Hirose, K.; Orlandi, R.; Léguillon, R.; Ogawa, T.; Soldner, T.; Hambsch, F.-J.; Astier, A.; Pollitt, A.; Petrache, C.; Tsekhanovich, I.; Mathieu, L.; Aïche, M.; Frost, R.; Czajkowski, S.; Guo, S.; Köster, U. Measurement of High-Energy Prompt Gamma-Rays from Neutron Induced Fission of U-235. EPJ Web Conf. 2017, 146, 04036.

(43) JEF Report 14: Table of Simple Integral Neutron Cross Section Data from JEF-2.2, ENDF/B-VI, JENDL-3.2, BROND-2 and CENDL-2. http://www.oecd-nea.org/dbdata/nds_jefreports/ (accessed June 3, 2020).

(44) Andor's iKon-L High Dynamic Range CCD Camera. https:// andor.oxinst.com/products/ikon-xl-and-ikon-large-ccd-series/ikon-l936 (accessed June 30, 2020).

(45) Schindelin, J.; Arganda-Carreras, I.; Frise, E.; Kaynig, V.; Longair, M.; Pietzsch, T.; Preibisch, S.; Rueden, C.; Saalfeld, S.; Schmid, B.; Tinevez, J.-Y.; White, D. J.; Hartenstein, V.; Eliceiri, K.; Tomancak, P.; Cardona, A. Fiji: an Open-Source Platform for Biological-Image Analysis. Nat. Methods 2012, 9 (7), 676-682.

(46) Schneider, C. A.; Rasband, W. S.; Eliceiri, K. W. NIH Image to ImageJ: 25 Years of Image Analysis. Nat. Methods 2012, 9 (7), 671675. 